\title{
GEAR TRANSMISSION RATTLE: ASSESSMENT OF MESHING FORCES UNDER HYDRODYNAMIC LUBRICATION
}

\author{
A. Fernandez-Del-Rincon · A. Diez-Ibarbia · S. \\ Theodossiades
}

Received: date / Accepted: date

\begin{abstract}
The dynamic behavior of gear transmissions poses several challenges from the standpoint of design and requires the availability of more advanced models capable of simulating a wide range of operating conditions. In this paper, several formulations to represent efforts related to the lubricant in gear transmissions subjected to reduced torque levels has been assessed. Under these conditions, the lubrication regime is hydrodynamic and the dynamic behavior of the meshing contacts can happen in different scenarios depending on both the lubricant properties and operating conditions. Such problems are cumbersome in gear transmissions in which acoustic performance is a determining design factor, such as in car applications. In this regard, gear rattle is one of the subjects of concern by powertrain designers. In spite of several authors have approached this phenomena, the most recent interest is focused on the role played by the lubricant. The variety of fundamentals and aims of the developed models in this respect requires a better understanding of the effect taken into account by the different formulations in the accurate modeling of hydrodynamic lubrication in gears subjected to low torques. This is the reason why, in this work, several alternatives currently available in the literature to address the formulation of efforts in hydrodynamic regime was collected and presented. Such formulations were implemented in a transmission model previously developed by the authors which was used to simulate different operating conditions in order to assess the results obtained with each one of the considered formulations.
\end{abstract}

Keywords Gear Rattle · Hydrodynamic lubrication · Vibrations

\section{List of Terms}

EHL Elastohydrodynamic Lubrication

$H D L$ Hydrodynamic Lubrication

$V_{e}$ Entraining velocity $\left(\frac{u_{1}+u_{2}}{2}\right)$

$\dot{h}$ Fluid film thickness velocity

$\lambda$ Stribeck parameter $\left(\lambda=\frac{h}{R a}\right)$

$\rho$ Fluid density

$\rho_{e q}$ Equivalent Curvature Radius

$a$ Half-width of the contact in the $x$ direction

$b$ Gear Width

$h$ Fluid film thickness

$h_{c}$ Central fluid film thickness

$h_{\max }$ Fluid film thickness maximum value

$h_{\text {min }}$ Fluid film thickness minimum value

$p$ Fluid pressure distribution

$u_{1}$ Profile velocity of the pinion in the $x$ direction

A. Fernandez-Del-Rincon

Department of Structural and Mechanical Engineering. ETSIIT University of Cantabria

Avda. de los Castros s/n. 39005 Santander. Spain

Tel.: +34-942200936

Fax.: +34-942201853

E-mail: fernandra@unican.es 
$u_{2}$ Profile velocity of the driven gear in the $x$ direction

$\eta$ Dynamic Viscosity

\section{Introduction}

Gearboxes are used to transmit the power from the engine or motor to the wheels in automotive Industry $[7,15,20,21]$. Nevertheless, they are not only used for this specific application but in aeronautic [3], energy $[19,22]$, agricultural sectors [16], among others. In gearboxes, there is a gear/stage, which is called active, that transmit the power from the input to the output shaft. Moreover, it exists other stages which are in contact but their objective is not to transmit the energy (low-loaded operating conditions), also denominated inactivate gears. In this regard, the role played by the lubricant in these gear pairs through its impact on the determination of dynamic loads in the contact areas is a crucial aspect and has been identified by the research community since the early works. Despite this recognition, accurate modeling of different damping mechanisms linked with the lubricant has received little attention in contrast to the large number of studies that have addressed the study of the dynamic behavior [1, 18,23,26].

This work is framed in this context and is aimed at assessing the role of the lubricating fluid into the operation of gear transmissions subjected to low torque levels. In the case of vehicle transmissions, this is a common situation that gives rise to the so-called "rattle" [25]. This phenomenon occurs as a result of repeated contacts between the flanks and the counter-flanks of the inactive gear pairs, which are subjected to torque and speed disturbances due to the engine dynamics. Furthermore, gear rattle turns into a lack of passenger comfort (noise) as well as is harmful, especially for the elements which support and are in contact with the shafts due to the generated and transmitted vibrations.

Under these conditions, the torque applied on the gear pair is due to the efforts related to tooth profile friction sliding (that in this preliminary work was neglected) and essentially due to the oil bearings drag. As a result, gear meshing forces have a reduced magnitude so that the lubrication regime in the tooth contact is fundamentally hydrodynamic $(H D L)$. To identify among regimes of lubrication, some parameters have been developed by the research community, being two of the most commonly used the Greenwood parameters and the Stribeck's one $(\lambda)$, which is the ratio between the fluid film thickness and the roughness of the teeth profile $\left(\lambda=h / R_{a}\right)[4,5]$. In this work, these parameters were utilized to assure that the transmission was on $H D L$ regime. As a consequence of having a hydrodynamic lubrication in the conjunction, it can be assumed that the surface deformation of the contact bodies is negligible and the lubricant properties remain unchanged in the vicinity of the conjunction [5]. These assumptions lead to a simplification of the Reynolds equation, which is the basis for the calculation of the pressure distribution of the contact and therefore of the hydrodynamic force. Specifically, two effects are usually considered to obtain the pressure in the conjunction, the fluid wedge and the fluid squeeze. The former is due to the profile shape and fluid entraining velocity and the latter is due to the approximation velocity between tooth profiles.

Depending on whether both effects are considered or not, it exists some formulations in order to calculate the hydrodynamic forces [12]. As a matter of fact, in literature, there are different studies that address the simulation of transmissions in these conditions, but surprisingly the proposed models show some dispersion. For this reason, in this study, several representative alternatives have been considered that hereinafter are to be designed as Rahnejat [26,27], Brancati [2], Gill-Jeong [13] and Guilbault [14]. The purpose of this study is to assess the available alternatives through its implementation in an enhanced model of gear transmission previously developed by the authors [11]. Among other capabilities, this model takes into account simultaneously the meshing efforts on both tooth sides, the variation of curvature radius through the meshing contact and also allows the introduction of gear profile [10] and indexing errors [9] which can affect the rattle phenomena.

The lubricant force formulations were reorganized in order to facilitate its implementation in the original model and subsequently applied to a gear transmission example which was used to simulate different operating conditions, in order to compare the response obtained with the different formulations and to assess the forces due to lubricant in hydrodynamic conditions. In the following, the structure of the manuscript is briefly described. After the introduction, in the second section, it is presented the procedure for obtaining each of the formulations considered in the study. Then, the gear transmission model, its main parameters and the characteristics that define simulation scenarios are described. Afterwards, the results, which has been obtained in the cases of study by using the different formulations, are presented. Finally, the main conclusions are described. 


\section{Fundamentals of hydrodynamic lubrication}

In order to avoid power losses, dry contact and overheating, the use of lubricants is common in gear transmissions. In lubrication theory, depending on the transmitted load, rheological properties and the gear roughness, four different regimes of lubrication are usually distinguished: Viscous elastic also called Elasto-Hydrodynamic Lubrication $(E H L)$, viscous rigid, iso-viscous elastic and iso-viscous rigid known as Hydrodynamic Lubrication $(H D L)$ [8]. In gearboxes, the active stage is generally under $E H L$ and the inactive ones are under $H D L$. As this work is focused on gear rattle, the latter are analysed in deep.

As commented, in order to assure that the transmission was on $H D L$, the Greenwood parameters and the Stribeck's one $(\lambda)$ were used in this work. Once the lubrication regime was determined, the fluid effect on the normal contact between tooth profiles was calculated. So as to represent its behaviour, most of the authors performs the Kelvin-Voigt's model, which means that the lubricant force in the conjunction is defined by a lineal viscous damping (equation 1 ).

$$
F_{C}=C_{l u b} \dot{\delta}
$$

So as to calculate this viscous damping and to assess the effects taken into account, it was necessary to solve the Reynolds equation. As the regime of lubrication is $H D L$, the rheological properties of the lubricant can be considered to remain constant in the conjunction vicinity. Moreover, as the contact length is in the order of a few microns [6], the sum of the profile velocities is approximately constant. Including these two approximations, Reynolds equation is simplified to the form presented in equation 2 (Figure 1 and 2).

$$
\underbrace{\frac{\rho}{12 \eta} \frac{\partial}{\partial t}\left(h^{3} \frac{\partial p}{\partial x}\right)}_{\text {Poiseulle }}=\underbrace{\rho \frac{\left(u_{1}+u_{2}\right)}{2} \frac{\partial h}{\partial x}}_{\text {Wedge }}+\underbrace{\rho \frac{\partial h}{\partial t}}_{\text {Squeeze }}=\underbrace{\rho \frac{\partial h}{\partial x} V_{e}}_{\text {Wedge }}+\underbrace{\rho h}_{\text {Squeeze }}
$$

Where $p$ is the fluid pressure distribution, $\eta$ the oil dynamic viscosity, $\rho$ the density, $h$ the film thickness, $\dot{h}$ the fluid film thickness velocity, $V_{e}$ the fluid entraining velocity and $u_{1}$ and $u_{2}$ are the profile velocities in the $x$ direction of the pinion and driven wheel (tangential to the teeth profile).

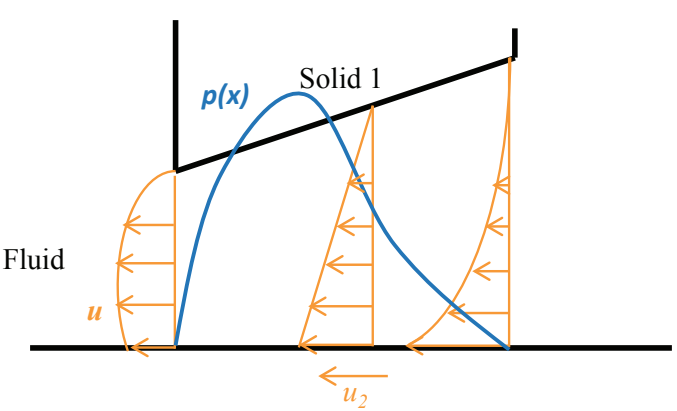

Solid 2

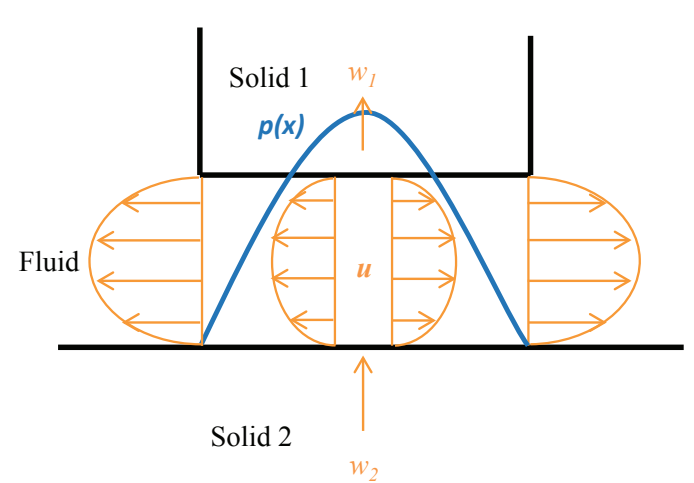

(b) Fluid velocity and pressure distribution when squeeze

(a) Fluid velocity and pressure distribution when wedge (b) Fluid velocity
term is considered
effect is considered

Fig. 1: Physical behavior of Reynolds equation terms considered to calculate the pressure distribution

The calculation of hydrodynamic forces requires obtaining the pressure distribution in the contact area, for which it is necessary to integrate the Reynolds equation. Depending on the boundary conditions and the simplifications considered, there are several solutions available in the literature. Two phenomena are involved, the wedge term (Figure 1(a)), which is associated with the oil inlet and outlet into the contact area, and the squeeze effect of the oil trapped between normal approaching surfaces (Figure 1(b)). In this regard, Gill-Jeong and Rahnejat formulations' consider both phenomena whilst Guilbault and Brancati ones' only take into account the squeeze effect regardless of the wedge term. Furthermore this consideration, the authors usually diverge in two main aspects: i) the procedure to obtain the pressure distribution; and ii) the extension of the contact area to be considered. Regarding the first aspect, the formulations differ in the shape of the pressure distribution and the boundary conditions considered during integration. With respect 
to the extension of the contact area, some authors consider that it is infinite, whilst others consider a finite value which depends on geometric parameters such as tooth addendum or equivalent radius of curvature.

Integration of equation 2 requires the definition of the fluid film thickness $(h)$ along the $x$ axis. In the case of spur gears, assuming ideal tooth profile, this contact takes place along a line extended across the tooth face width. Under these conditions, known the central fluid film thickness $\left(h_{c}\right)$, the fluid thickness of a gear profile cross-section can be represented by Taylor series expansion as a function of the equivalent radius of curvature $\left(\rho_{e q}\right)$ according to:

$$
h=h_{c}+\frac{x^{2}}{2 \rho_{e q}}
$$

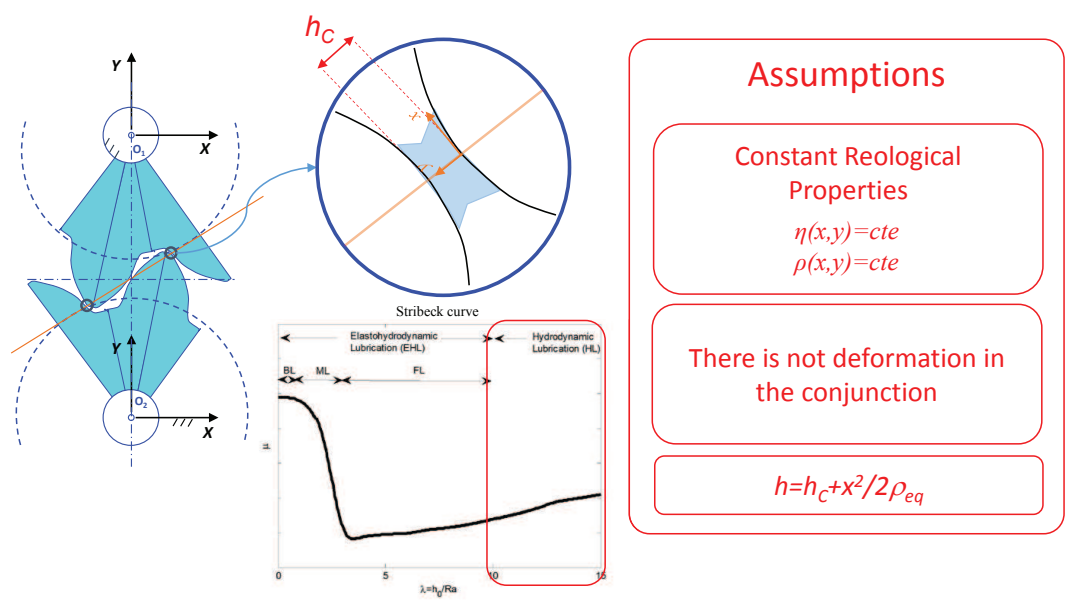

Fig. 2: Summarize of the approximations generally considered in $H D L$

With this consideration, the force due to the lubricant in its general form can be calculated by the integration along the gear face width $(b)$ in the considered domain $\left(x_{0}<x<x_{1}\right)$ :

$$
F_{\text {lub } b_{H L}}=b \int_{x_{0}}^{x_{1}} p(x) d x=F_{W e d g e}+F_{\text {Squeeze }}=C_{e q}^{W e d g e} V_{e}-C_{e q}^{\text {Squeeze }} \dot{h}
$$

It can be observed that depending of the limits of the integral and the effects considered (wedge and squeeze phenomena), different solution of the hydrodynamic force is obtained. In this regard, there are formulations which do not consider the wedge term [2,14], whilst others do [13,27]. In the following, a description of the approaches of contact forces in hydrodynamic regime that were assessed in this work is presented.

\subsection{Rahnejat et al. formulation}

Rahnejat considered individually Wedge and Squeeze terms in equation 2. When the Squeeze term is neglected, the solution is obtained using the Half-Sommerfeld boundary conditions, which impose the location of a maximum at $x_{a}$ (equation 5 ).

$$
\left.\begin{array}{ll}
\frac{\partial p}{\partial x}=0 & \rightarrow x=-x_{a} \\
p=0 & \rightarrow x=-\infty
\end{array}\right\} \quad p(x)=\frac{6 \eta \sqrt{2 \rho_{e q}}}{h_{c}^{\frac{3}{2}}} p(\bar{x}) V_{e}
$$

Where $p(\bar{x})$ is the dimensionless pressure distribution which can be found in [24] and an example of its shape is presented in Figure 3(a).

Then, the hydrodynamic force is calculated by integrating the pressure distribution between $\pm \infty$, leading to the following expression: 


$$
F_{H D L}^{W e d g e}=b \int_{-\infty}^{\infty} p(x) d x=2 b \eta \frac{\rho_{e q}}{h_{c}} V_{e}
$$

However, when the Wedge term is removed, a quadratic pressure distribution is taken into account, where the imposed boundary conditions are that pressure reaches zero value at $\pm \infty$. This results in a symmetric pressure distribution without negative values (Figure 3(b) and equation 7).

$$
\left.\begin{array}{ll}
\frac{\partial p}{\partial x}=0 & \rightarrow x=0 \\
p=0 & \rightarrow x= \pm \infty
\end{array}\right\} \quad p(x)=2 \eta h_{c} \frac{1}{\left(x^{2}+2 \rho_{e q} h_{c}\right)^{2}} \dot{h}
$$

Integrating the pressure distribution between $\pm \infty$, the hydrodynamic force is obtained by:

$$
F_{H D L}^{\text {Squeeze }}=b \int_{-\infty}^{\infty} p(x) d x=\frac{3 \pi b \eta}{\sqrt{2}}\left(\frac{\rho_{e q}}{h_{c}}\right)^{\frac{3}{2}} \dot{h}
$$
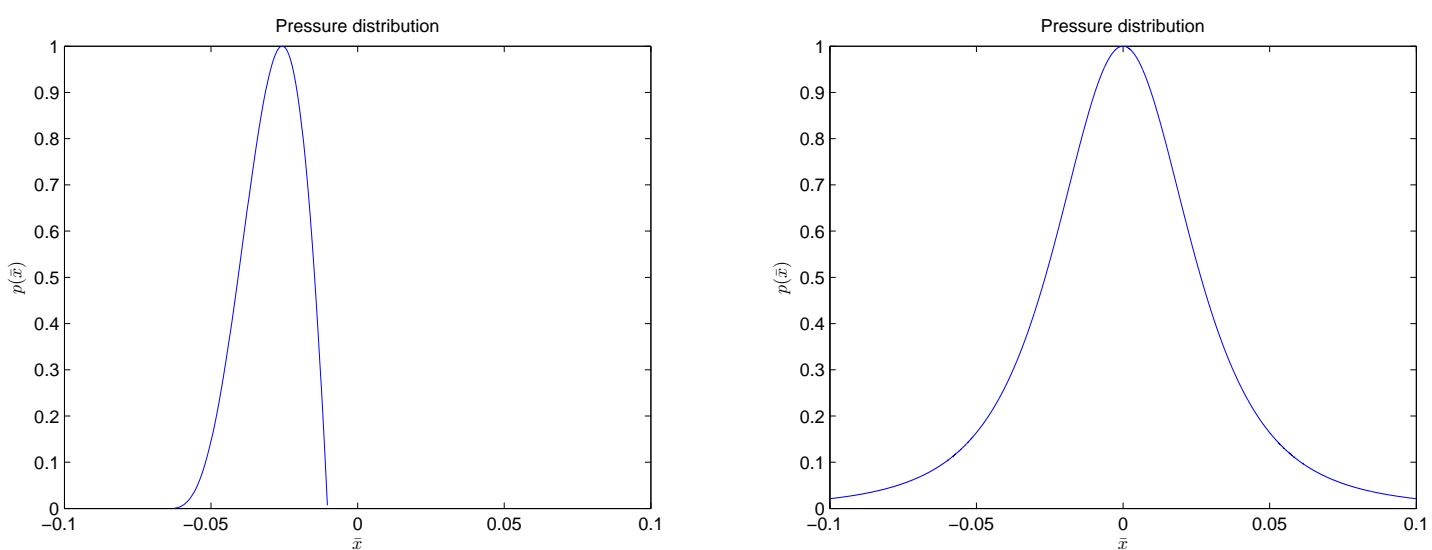

(a) Dimensionless pressure distribution when Half- (b) Dimensionless pressure distribution when pure squeeze Sommerfeld conditions are considered motion is considered

Fig. 3: Example of the pressure distribution used by Rahnejat's formulation

Superposing the solutions for each case (equation 6 and 8), the Rahnejat's expression is obtained (equation 9).

$$
F_{H D L}= \begin{cases}2 b \eta \frac{\rho_{e q}}{h_{c}} V_{e}-\frac{3 \pi b \eta}{\sqrt{2}}\left(\frac{\rho_{e q}}{h_{c}}\right)^{\frac{3}{2}} \dot{h} & \dot{h}<0 \\ 2 b \eta \frac{\rho_{e q}}{h_{c}} V_{e} & \dot{h} \geq 0\end{cases}
$$

The term $\dot{h}=\frac{\partial h}{\partial t}$ represents the speed when the contact profiles are approaching or moving away, being negative when they are approaching. According to equation 9, two terms are involved when the profiles are approaching $(\dot{h}<0)$. The first is due to the Wedge term, whilst the second regards the fluid Squeeze effect. On the other hand, when the profiles are moving away $(\dot{h}>0)$, only the Wedge term is taken into consideration.

\subsection{Brancati et al. formulation}

In contrast to the procedure described in the preceding subsection 2.1, Brancati et al. disregard the Wedge term in equation 2, assuming that the pressure distribution is symmetrical with respect to the maximum pressure point located at $x=0$ and imposing a zero value of the pressure at $\pm a$ as presented in equation 10 and Figure 4. 


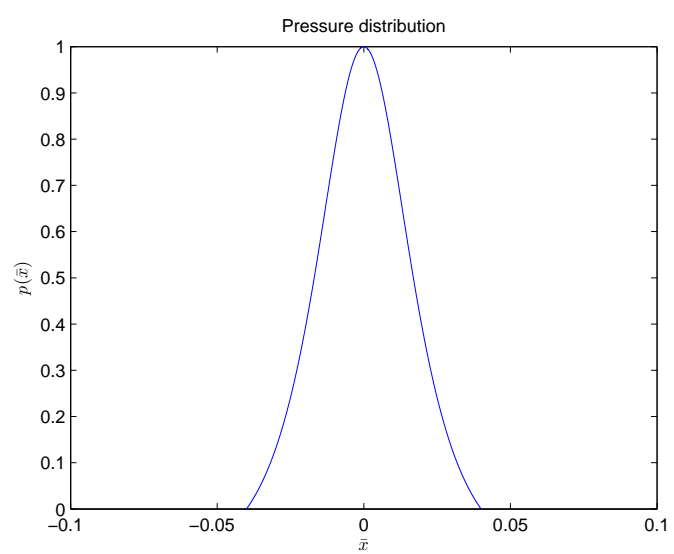

Fig. 4: Example of the pressure distribution used by Bracati's formulation

$$
\left.\begin{array}{ll}
\frac{\partial p}{\partial x}=0 & \rightarrow x=0 \\
p=0 & \rightarrow x= \pm a
\end{array}\right\} \quad p(x)=3 \eta \rho_{e q}^{3}\left[\frac{1}{\left(a^{2}+\rho_{e q} h_{c}\right)^{2}}-\frac{1}{\left(x^{2}+\rho_{e q} h_{c}\right)^{2}}\right] \dot{h}
$$

Once the pressure distribution is obtained, the hydrodynamic force is determined by integrating it along the contact area, which in the $x$ direction is between $\pm a$, leading to the expression 11:

$$
F_{H D L}=b \int_{-a}^{a} p(x) d x=3 \eta b\left(\frac{\rho_{e q}}{h_{c}}\right)^{\frac{3}{2}}\left[\frac{a\left(a^{2}-\rho_{e q} h_{c}\right) \sqrt{\rho_{e q} h_{c}}+\left(a^{2}+\rho_{e q} h_{c}\right)^{2} \arctan \left(\frac{a}{\sqrt{\rho_{e q} h_{c}}}\right)}{\left(a^{2}+\rho_{e q} h_{c}\right)^{2}}\right] \dot{h}
$$

Where $a$ represents the half-width of the contact affected by the presence of lubricant and is usually determined arbitrarily depending on the amount of lubricant and on the dimensions of the teeth. The authors do not indicate whether the expression 11 must be annulled when the profiles are moving away.

\subsection{Gill-Jeong formulation}

Unlike Rahnejat's model, Gill-Jeong calculates pressure distribution considering both terms of the simplified Reynolds equation 2. A symmetric distribution of pressure is assumed, imposing the same boundary conditions as Brancati (equation 12 and Figure 5).

$$
\begin{aligned}
& p(x)=3 \eta \rho_{e q}^{3}\left[\frac{1}{\left(a^{2}+\rho_{e q} h_{c}\right)^{2}}-\frac{1}{\left(x^{2}+\rho_{e q} h_{c}\right)^{2}}\right] \dot{h} \\
& \left.\begin{array}{ll}
\frac{\partial p}{\partial x}=0 & \rightarrow x=0 \\
p=0 & \rightarrow x= \pm a
\end{array}\right\} \quad+\frac{3 \eta \rho_{e q}}{2 h_{c}}\left[\frac{a\left(a^{2}-\rho_{e q} h_{c}\right)}{\left(a^{2}+\rho_{e q} h_{c}\right)^{2}}+\frac{x\left(x^{2}-\rho_{e q} h_{c}\right)}{\left(x^{2}+\rho_{e q} h_{c}\right)^{2}}\right] V_{e} \\
& +\frac{3 \eta \rho_{e q}}{2 h_{c}}\left[\frac{1}{\sqrt{\rho_{e q} h_{c}}}\left\{\arctan \left(\frac{a}{\sqrt{\rho_{e q} h_{c}}}+\arctan \left(\frac{x}{\sqrt{\rho_{e q} h_{c}}}\right)\right)\right\}\right] V_{e}
\end{aligned}
$$

The resulting pressure distribution is integrated in the distance range of $-a<x<a$, proposing the equation 13:

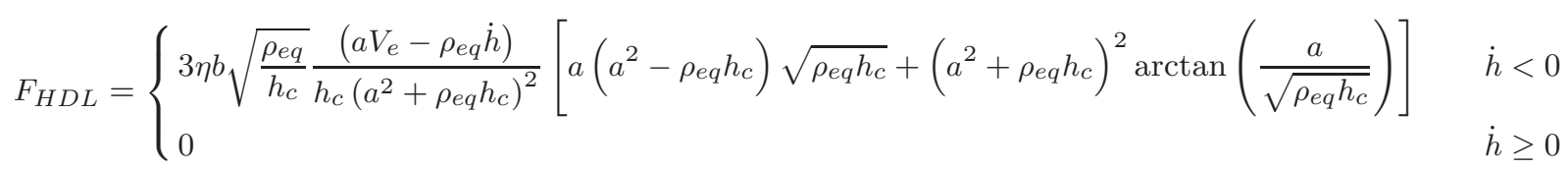




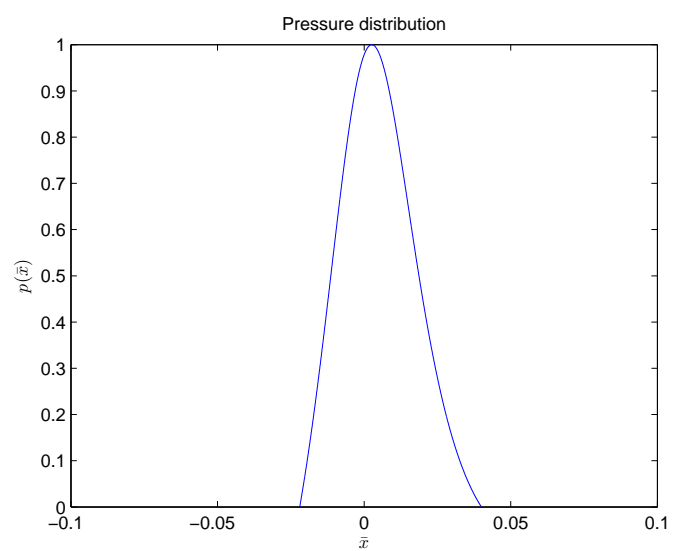

Fig. 5: Example of the pressure distribution used by Gill-Jeong's formulation

It is interesting to point out that Gill-Jeong suggests that the hydrodynamic force should be canceled when a separation of the tooth profiles contact occurs $(\dot{h} \geq 0)$. This approach contrasts with the Rahnejat's proposal, in which only the term linked to the lubricant squeeze is disregarded.

\subsection{Guilbault et al. formulation}

Guilbault, Lalonde and Thomas neglected the entraining lubricant effect and integrate equation 2 imposing as boundary conditions that $p( \pm \infty)=0$ (equation 14). Nevertheless, to obtain the resultant force they integrate the resulting pressure distribution in the distance range of $-a<x<a$, reaching equation 15:

$$
\begin{gathered}
\left.\begin{array}{cc}
\frac{\partial p}{\partial x}=0 & \rightarrow x=0 \\
p=0 & \rightarrow x= \pm \infty
\end{array}\right\} \quad p(x)=\frac{12 \eta \rho_{e q}}{\left(x^{2}+2 \rho_{e q} h_{c}\right)^{2}} \dot{h} \\
F_{H D L}=2 b \eta\left[3 \sqrt{2}\left(\frac{\rho_{e q}}{h_{c}}\right)^{\frac{3}{2}} \arctan \left(\frac{a}{\sqrt{2 \rho_{e q} h_{c}}}\right)+\frac{6 a \rho_{e q}^{2}}{h_{c}\left(a^{2}+2 \rho_{e q} h_{c}\right)}\right] \dot{h}
\end{gathered}
$$

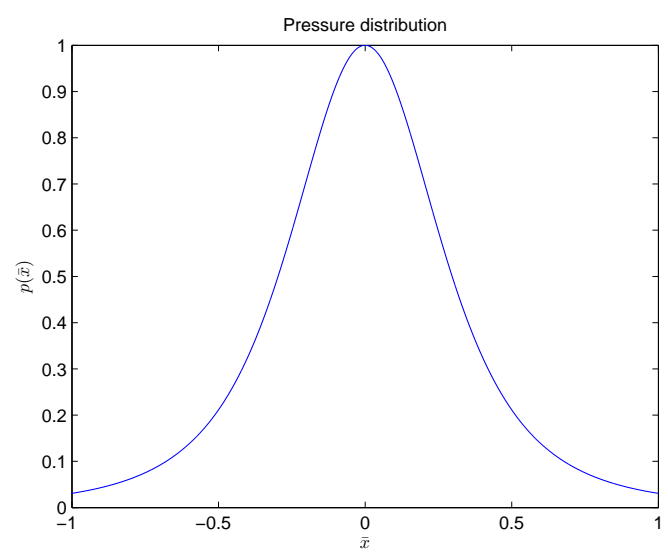

Fig. 6: Example of the pressure distribution used by Guilbault's formulation

Where $a$ is considered variable along the contact and equals to the lowest value between the radius of the contacting point and the tip radius. As in Brancati's formulation, the force is not constrained to be null when the profiles are moving away. 


\section{Dynamic model description}

In order to assess the characteristics of the formulations described in section 2, they were implemented in the gear transmission model previously developed by the authors [9-11]. The original model took into account a total of nineteen degrees of freedom (d.o.f.) but in this application, it was reduced to two rotational d.o.f. (Figure 7$)\left(\theta_{1 R 1}\right.$ and $\left.\theta_{2 R 1}\right)$ linked to each wheel, resulting in the system of equations 16.

$$
\left\{\begin{array}{l}
J_{1 R 1} \ddot{\theta}_{1 R 1}+f_{1 R 1 \theta}\left(\theta_{1 R 1}, \theta_{2 R 1}, \dot{\theta}_{1 R 1}, \dot{\theta}_{2 R 1}\right)=T_{1 R 1} \\
J_{2 R 1} \ddot{\theta}_{2 R 1}+f_{2 R 1 \theta}\left(\theta_{1 R 1}, \theta_{2 R 1}, \dot{\theta}_{1 R 1}, \dot{\theta}_{2 R 1}\right)=T_{2 R 1}
\end{array}\right.
$$

Where $J_{j R k}$ is the inertia of the wheel $k$ located in the shaft $j, \theta_{j R k}$ is the rotational degree of freedom linked to $J_{j R k}$ inertia, $f_{j R k \theta}$ represents the torque due to the contact forces and $T_{j R k}$ represents the external torque applied to the wheel $k$ located in the shaft $j$.

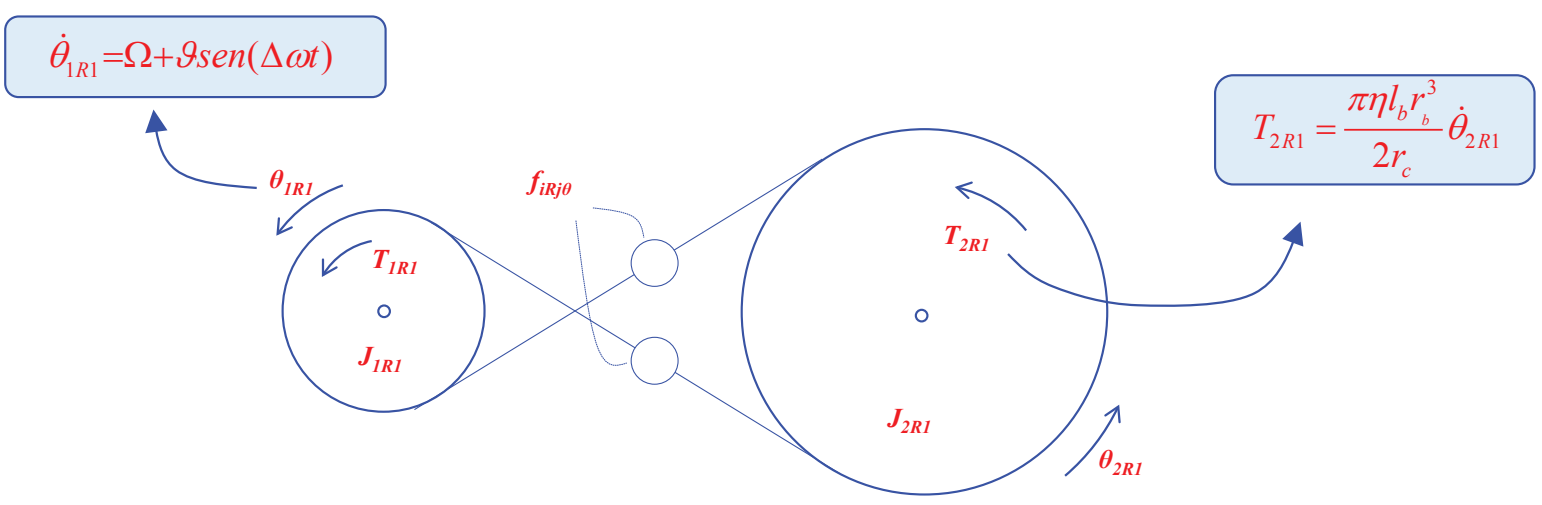

Fig. 7: Dynamic model schema

The meshing forces $f_{j R k \theta}$ are determined by identifying and calculating the distances $\delta_{i}$ (being $i$ the number referred to each contact) between pairs of teeth potentially in contact, taking into account the contact on both tooth sides. Subsequently, the resulting forces are reduced to the center of each wheel as an equivalent torque [11].

The introduction of hydrodynamic forces requires a modification of the original model. Thus, each contact force $\left(F_{i}\right)$ is composed of two different terms (see equation 17).

$$
F_{i}= \begin{cases}F_{\text {mesh }_{i}} & \delta_{i} \leq 0 \\ F_{H D L_{i}} & \delta_{i}>0\end{cases}
$$

The first occurs when the profile contact is closed, in other words, the minimum distance between them is negative $\left(\delta_{i} \leq 0\right)$. In this case the resultant force corresponds to the meshing force $\left(F_{m e s h_{i}}\right)$ which is determined by multiplying the magnitude of interference (overlap) by the stiffness corresponding to the contact obtained from a previous quasi-static analysis [10]. Conversely, when the contact between profiles is not closed (then $h_{i}=\delta_{i}$ ), the force acting is the hydrodynamic one due to lubricant $\left(F_{H D L_{i}}\right)$ which is obtained from the corresponding formulation. This procedure is summarized in Figure 8.

In tribodynamic models found in the literature [14,17], from $h_{\min }$ to the contact, there is a transition where the forces due to the lubricant are generally calculated applying $E H L$ fundamentals. Although the assumption of considering hydrodynamic forces lead to an error, this error is acceptable in gear rattle conditions since the applied force is very low.

For brevity sake, details regarding the procedure for calculating the meshing forces when the contacts are closed $\left(F_{\text {mesh }}\right)$ are not provided. The reader interested in further explanation is referred to [9-11].

In order to obtain the hydrodynamic forces $\left(F_{H D L_{i}}\right)$, the expressions presented in section 2 were implemented and reorganized in the form:

$$
F_{H D L_{i}}=\left\{\begin{array}{ll}
C_{e q_{i}}^{W e d g e} V_{e_{i}}-C_{e q_{i}}^{\text {Squeeze }} \dot{h_{i}} & \dot{h_{i}}<0 \\
C_{e q_{i}}^{W e d g e} V_{e_{i}} & \dot{h_{i}} \geq 0
\end{array}\right\}=b \eta \begin{cases}A_{i} V_{e_{i}}-B_{i}\left(\frac{\rho_{e q_{i}}}{h_{c_{i}}}\right)^{\frac{3}{2}} \dot{h_{i}} & \dot{h_{i}}<0 \\
A_{i} V_{e_{i}} & \dot{h_{i}} \geq 0\end{cases}
$$




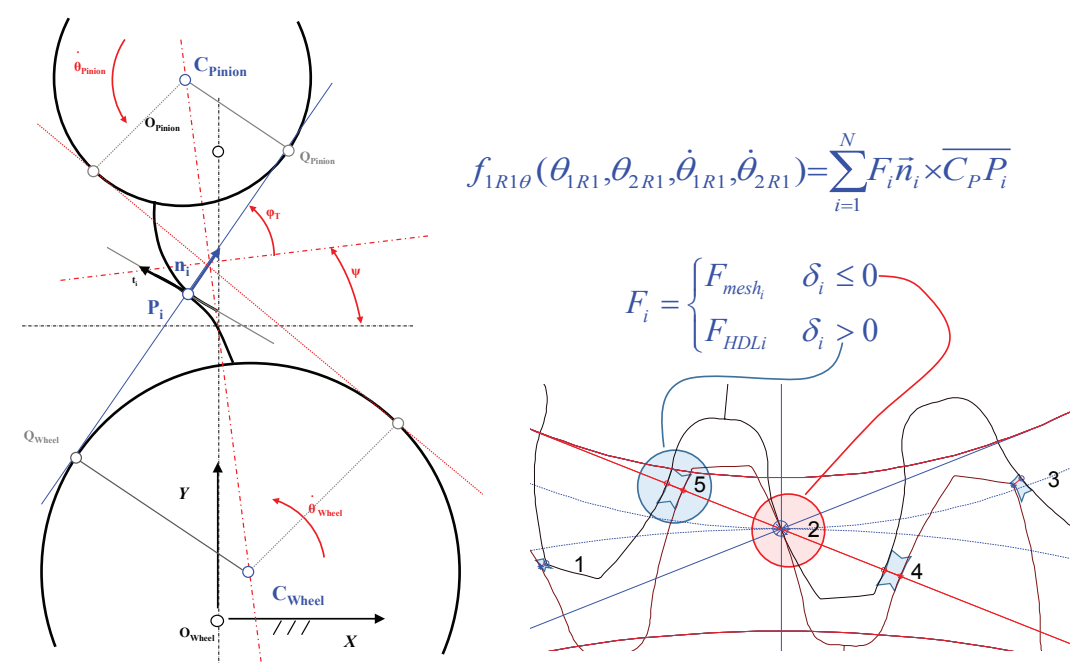

Fig. 8: Schema of the force algorithm choice

As can be seen, the forces due to the squeeze effect are only considered when the profiles are approaching $(\dot{h}<0)$, being neglected when they are moving away, even if the authors take them into account. Tables 1 and 2 list the implemented expressions for each term $A_{i}$ and $B_{i}$ of equation 18.

Table 1: $A_{i}$ expressions for hydrodynamic force

\begin{tabular}{cc}
\hline Formula & $A_{i}$ \\
\hline Rahnejat & $2 \frac{\rho_{e q}}{h_{c}}$ \\
Brancati & 0 \\
Gill-Jeong & $3 a \frac{\rho_{e q}^{1 / 2}}{h_{c}^{3 / 2}}\left[\frac{a\left(a^{2}-\rho_{e q} h_{c}\right) \sqrt{\rho_{e q} h_{c}}+\left(a^{2}+\rho_{e q} h_{c}\right)^{2} \arctan \left(\frac{a}{\sqrt{\rho_{e q} h_{c}}}\right)}{\left(a^{2}+\rho_{e q} h_{c}\right)^{2}}\right]$ \\
Guilbault & 0 \\
\hline
\end{tabular}

Table 2: $B_{i}$ expressions for hydrodynamic force

\begin{tabular}{lr}
\hline Formula & \\
\hline Rahnejat & $3\left[\frac{a\left(a^{2}-\rho_{e q} h_{c}\right) \sqrt{\rho_{e q} h_{c}}+\left(a^{2}+\rho_{e q} h_{c}\right)^{2} \arctan \left(\frac{a}{\sqrt{\rho_{e q} h_{c}}}\right)}{\left(a^{2}+\rho_{e q} h_{c}\right)^{2}}\right]$ \\
Brancati & $3\left[\frac{a\left(a^{2}-\rho_{e q} h_{c}\right) \sqrt{\rho_{e q} h_{c}}+\left(a^{2}+\rho_{e q} h_{c}\right)^{2} \arctan \left(\frac{a}{\sqrt{\rho_{e q} h_{c}}}\right)}{\left(a^{2}+\rho_{e q} h_{c}\right)^{2}}\right]$ \\
Gill-Jeong & $3\left[\frac{4 a\left(a^{2}+2 \rho_{e q} h_{c}\right) \sqrt{\rho_{e q} h_{c}}+2 \sqrt{2}\left(a^{2}+2 \rho_{e q} h_{c}\right)^{2} \arctan \left(\frac{a}{\sqrt{2 \rho_{e q} h_{c}}}\right)}{\left(a^{2}+2 \rho_{e q} h_{c}\right)^{2}}\right]$ \\
\hline
\end{tabular}

It should be noted that the distance between the profiles $h_{c}$ in the denominator involves an infinite value of the hydrodynamic force when the distance between the profiles tends to be null. To avoid this singularity, a saturation value $\left(h_{\min }\right)$ was used when the distance $h$ was smaller than this value. On the other hand, it was also adopted a maximum distance value $\left(h_{\max }\right)$ from which the hydrodynamic force is considered negligible (Figure 9).

The model described above was applied to simulate the dynamic behavior of a transmission defined by the parameters shown in Table 3.

Moreover, the resistant torque on the driven wheel was formulated as a torsional viscous damper dependant of the journal bearing parameters, according to equation 19 [27]. 


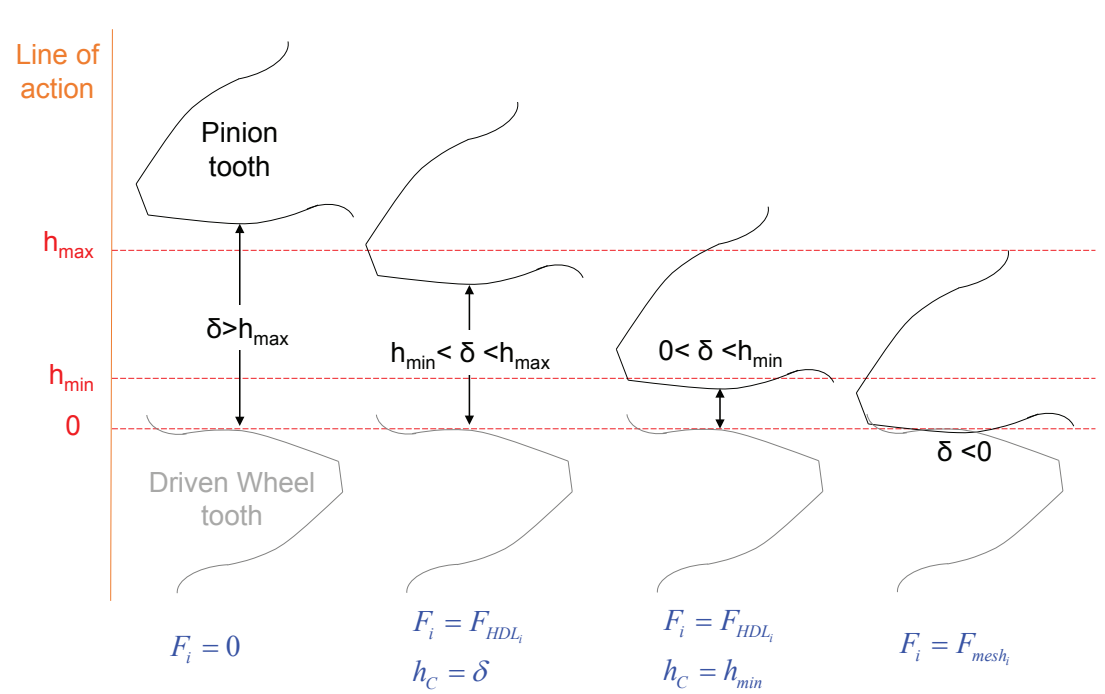

Fig. 9: Schema of the force algorithm choice

Table 3: Gear transmission parameters

\begin{tabular}{cccc}
\hline Parameter & Value & Parameter & Value \\
\hline Pinion teeth $\left(z_{1}\right)$ & 18 & Rack Addendum $\left(A d_{\text {rack }}\right)$ & $1.25 \mathrm{~m}$ \\
Wheel teeth $\left(z_{2}\right)$ & 36 & Rack Deddendum $\left(D d_{\text {rack }}\right)$ & $1 \mathrm{~m}$ \\
Pinion inertia $\left(J_{1 R 1}\right)\left[\mathrm{kgm}^{2}\right]$ & $1.75 \mathrm{e}-4$ & Rack tip radius $\left(r_{t}\right)$ & $0.25 \mathrm{~m}$ \\
Wheel inertia $\left(J_{2 R 1}\right)\left[\mathrm{kgm}^{2}\right]$ & $2.8 \mathrm{e}-3$ & Face width $(b)[\mathrm{mm}]$ & 26.7 \\
Module $(\mathrm{m})[\mathrm{mm}]$ & 3 & Oil viscosity $(\eta)[P a s]$ & 0.08 \\
Poisson coef. $(\nu)$ & 0.3 & Working distance $(d)[\mathrm{mm}]$ & 81.1 \\
Pressure angle $\left(\varphi_{i}\right)[$ degree $]$ & 20 & $h_{\text {min }} / h_{\text {max }}[\mathrm{mm}]$ & $8 \mathrm{e}-4 / 1$ \\
Journal bearing radius $\left(r_{b}\right)[\mathrm{mm}]$ & 10 & Journal bearing length $\left(l_{b}\right)[\mathrm{mm}]$ & 15 \\
Radial clearance $\left(r_{c}\right)[\mathrm{mm}]$ & 0.025 & Half-width contact $(a)[\mathrm{mm}]$ & 1 \\
\hline
\end{tabular}

$$
T_{2 R 1}=\frac{\pi \eta_{b} l_{b} r_{b}^{3}}{2 r_{c}} \dot{\theta}_{2 R 1}
$$

Where $\eta_{b}$ is the journal bearing dynamic viscosity (same oil as in the gears $\eta_{b}=\eta$ ), $r_{b}$ and $l_{b}$ are the nominal radius and width of the journal bearing and $r_{c}$ is the radial clearance.

The pinion angular velocity (equation 20) is imposed considering the superposition of an average value of angular velocity $(\Omega)$ and a harmonic excitation defined by its amplitude $(\vartheta)$ and frequency $(\Delta \omega)$. Three scenarios were considered depending on the values considered of the mean angular velocity, which are presented in Table 4 . Furthermore, the values of the velocity excitation $(\vartheta$ and $\Delta \omega)$ were chosen in order to maintain the angular acceleration constant among scenarios.

$$
\dot{\theta}_{1 R 1}=\Omega+\vartheta \sin \Delta \omega t
$$

Table 4: Values of the parameters which define the pinion angular velocity

\begin{tabular}{cccc}
\hline Scenario number & $\Omega[\mathrm{rpm}]$ & $\vartheta[\mathrm{Rad} / \mathrm{s}]$ & $\Delta \omega[\mathrm{Hz}]$ \\
\hline 1 & 0 & {$[10.472,5.236,2.618]$} & {$[4,8,16]$} \\
2 & 500 & {$[10.472,5.236,2.618]$} & {$[4,8,16]$} \\
3 & 1000 & {$[10.472,5.236,2.618]$} & {$[4,8,16]$} \\
\hline
\end{tabular}

\section{Results and discussion}

In Figure 10, the transmission error obtained in each considered scenario is presented. The horizontal lines (in dashed lines) represent the backlash, thus when the transmission error takes values outside this range, 
the profiles have physical interference. The reference position is considered when there is contact in the pitch point and this is the reason why the lower value of the backlash is zero.

When average velocity is null (only harmonic excitation is considered), all the approaches lead to contacts between both tooth sides (see Figure 11)). In this scenario, the effect of the entraining lubricant is less important than the Squeeze term and, as a consequence, it was expected that the different approaches should provide similar results. Nevertheless, there is a remarkable difference in the behaviour when GillJeong's formulation is used, although the squeeze term is identical to the Brancati's approach.

In contrast, Rahnejat's and Gill-Jeong's formulations do not lead to teeth contacts in scenarios 2 and 3 (and therefore no rattle), highlighting the importance of the lubricant entrance effect (Figures 11 and 12). Otherwise, Brancati's and Guilbault's formulations provide similar results and unlike the other approaches, there are contacts on both tooth sides. It can be also appreciated how the Brancati's approach leads to loss of contact between $0.8-1$ and 1.4-1.6 cycles with the highest frequency of angular excitation $(\delta \omega=16 \mathrm{~Hz})$.

Worthy of mention is also the remarkable oscillation observed in scenarios 2 and 3 particularly with GillJeong's formulation. These oscillations are due to meshing cycles corresponding to the average velocity of 500 and $1000 \mathrm{rpm}$. This aspect can also be appreciated although with less intensity when Rahnejat's formulation is applied. Gill-Jeong's approach does not seems to be valid for the operation conditions considered in the simulations, leading to results clearly different to those provided by Rahnejat's formulation which a priori should be expected to be similar. Since the Squeeze term is identical to the term provided by Brancati et al., it can be concluded that the Wedge component should be reviewed.

\section{Conclusions}

In this paper, several formulations to represent lubricant efforts in gear transmissions working under low torque levels (rattle conditions) were analysed. Based on the results obtained, it can be concluded that in this conditions, the effect of lubricant entrance in the contact area plays a decisive role in the dynamic behavior. As a result, the hydrodynamic forces obtained by formulations which consider this aspect (Rahnejat and GillJeong) are high enough to avoid contact between profiles when average speeds are applied. Moreover, GillJeong's approach provides results much more different from the other formulations. The deeper assessment of this formulation leads to the conclusion that when entraining velocity has a higher order of magnitude than squeeze velocity, the arc tangent of its formulation prevails over the squeeze term and gives results which do not match with the rest of formulae. Therefore, the Gill-Jeong's formulation is very responsive to speed changes which can lead to error in the rattle behavior modeling.

Although Brancati's and Guilbault's formulations provide good results depending on the application, the only assessed formulation to model gear rattle accurately is the Rahnejat's.

As a conclusion, simulation of gear rattle requires a careful choice of the approach used for calculation of hydrodynamic forces. The results presented in this paper should be considered as a preliminary work. In this line, more research is required in the future in order to assess more formulations which take into account entraining lubricant effect and the usefulness and reliability of the available approaches.

Acknowledgments This work has been supported by project DPI2013-44860 funded by the Spanish Ministry of Science and Technology, PRX14 / 00451 project funded by the Spanish Ministry of Education, Culture and Sports and COST Action TU 1105.

\section{References}

1. M. Ankouni, A.A. Lubrecht, and P. Velex. Modelling of damping in lubricated line contacts - applications to spur gear dynamic simulations. Proceedings of the Institution of Mechanical Engineers, Part C: Journal of Mechanical Engineering Science, 230(7-8):1222-1232, 2015.

2. R. Brancati, E. Rocca, and R. Russo. An analysis of the automotive driveline dynamic behaviour focusing on the influence of the oil squeeze effect on the idle rattle phenomenon. Journal of Sound and Vibration, 303(3-5):858-872, 2007.

3. F. Chaari, M.S. Abbes, F.V. Rueda, A.F. del Rincon, and M. Haddar. Analysis of planetary gear transmission in non-stationary operations. Frontiers of Mechanical Engineering, 8(1):88-94, 2013.

4. W. Chong and M. De La Cruz. Elastoplastic contact of rough surfaces: A line contact model for boundary regime of lubrication. Meccanica, 49(5):1177-1191, 2014.

5. M. De La Cruz, W. Chong, M. Teodorescu, S. Theodossiades, and H. Rahnejat. Transient mixed thermoelastohydrodynamic lubrication in multi-speed transmissions. Tribology International, 49:17-29, 2012.

6. M. De la Cruz, S. Theodossiades, H. Rahnejat, and P. Kelly. Analysis of non-linear impact dynamics in automotive transmissions: Gear rattle. In Proceedings of the 7th International Conference on Engineering Computational Technology, 2010. 


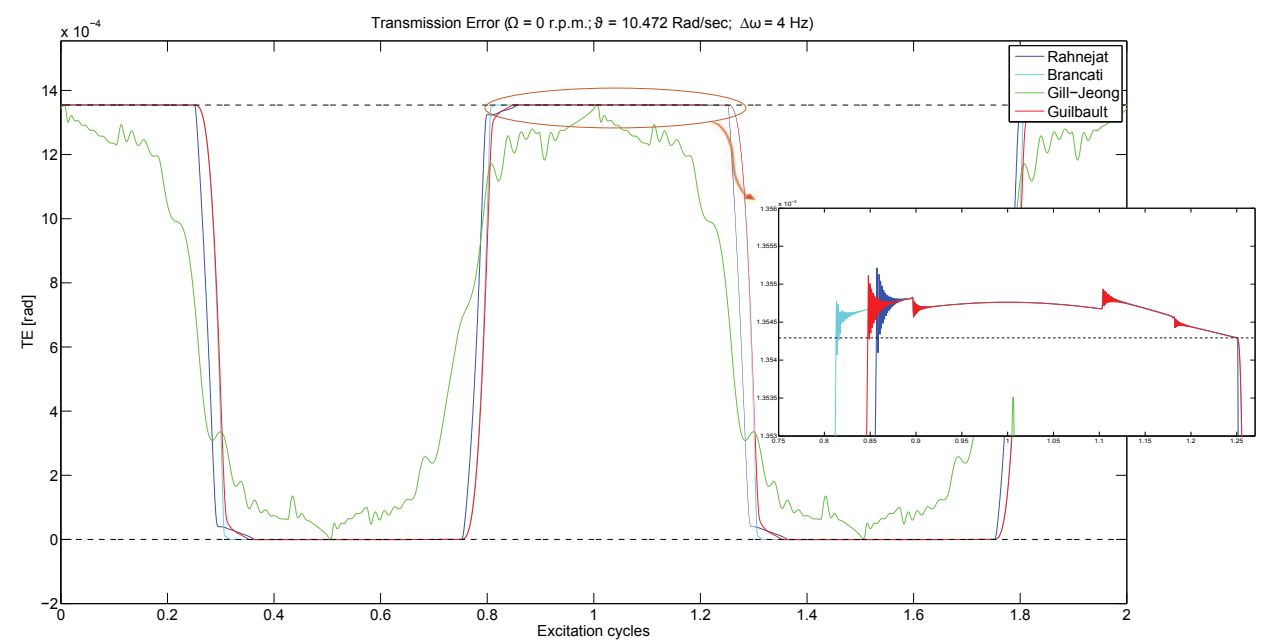

(a) Transmission error when $\Omega=0 \mathrm{rpm}, \vartheta=10.472 \mathrm{Rad} / \mathrm{s}$ and $\Delta \omega=4 \mathrm{~Hz}$

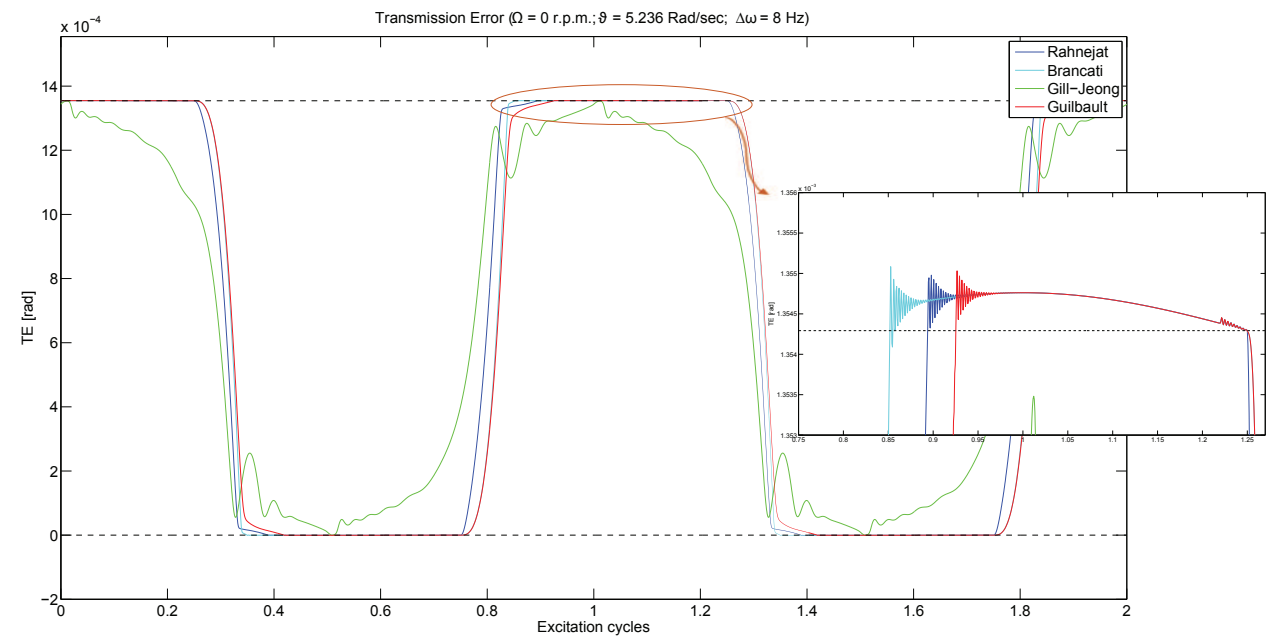

(b) Transmission error when $\Omega=0 \mathrm{rpm}, \vartheta=5.236 \mathrm{Rad} / \mathrm{s}$ and $\Delta \omega=8 \mathrm{~Hz}$

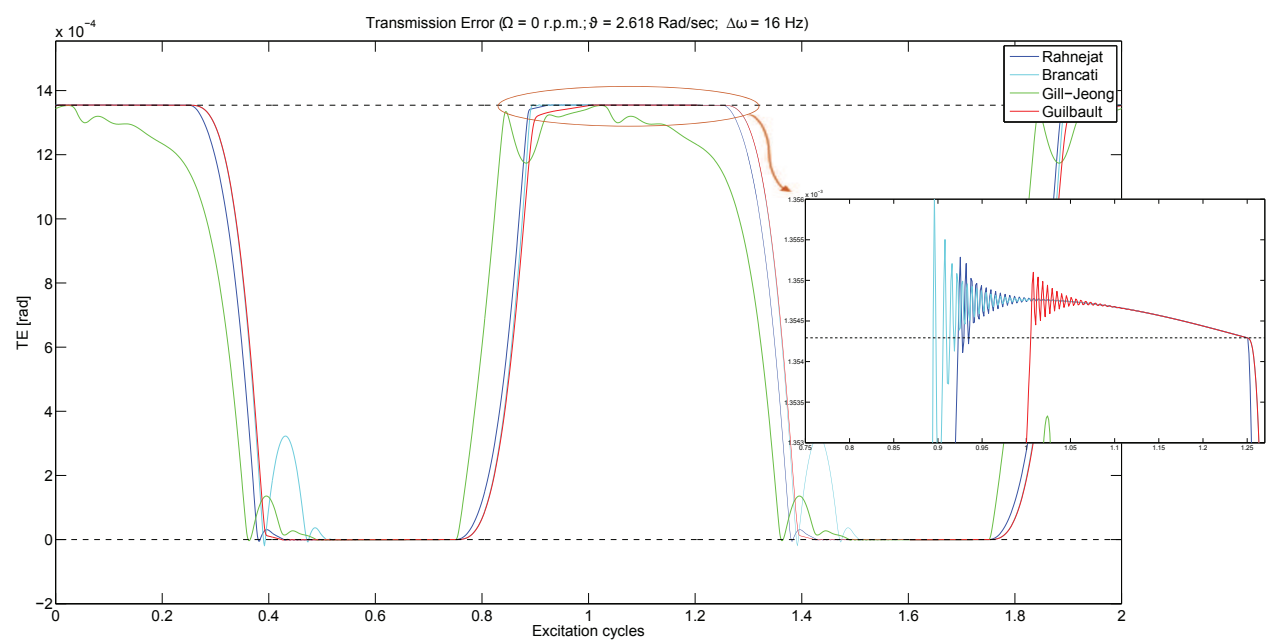

(c) Transmission error when $\Omega=0 \mathrm{rpm}, \vartheta=2.618 \mathrm{Rad} / \mathrm{s}$ and $\Delta \omega=16 \mathrm{~Hz}$

Fig. 10: Transmission error when there is not a mean velocity value for different harmonic excitations 


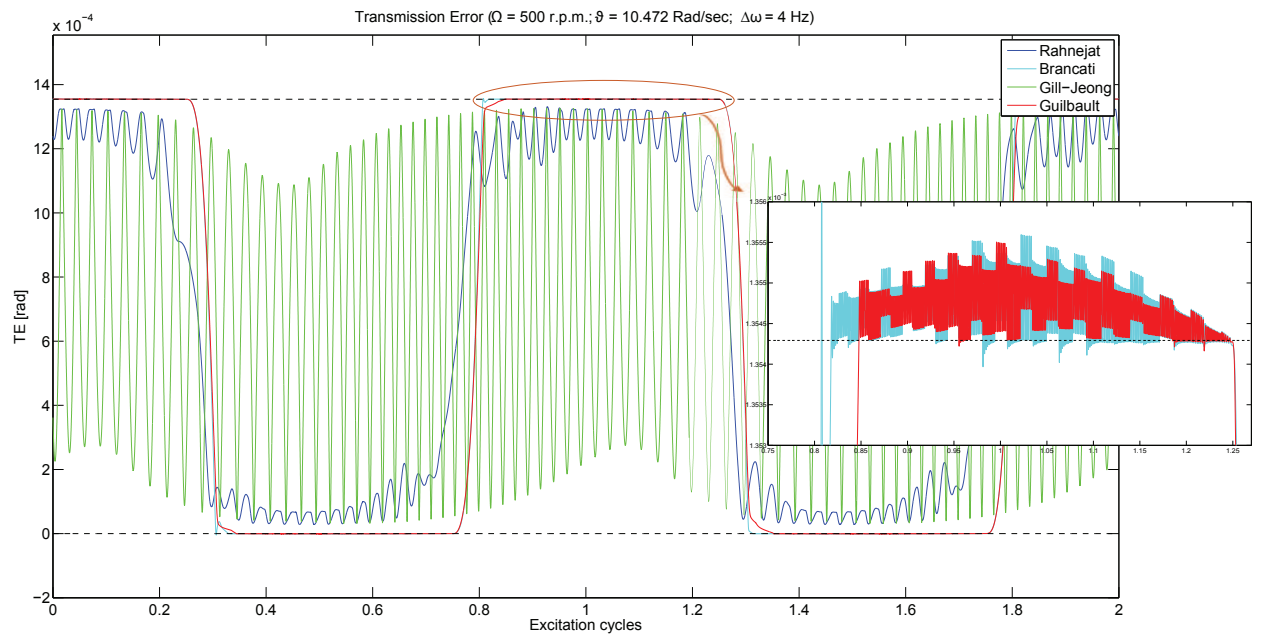

(a) Transmission error when $\Omega=500 \mathrm{rpm}, \vartheta=10.472 \mathrm{Rad} / \mathrm{s}$ and $\Delta \omega=4 \mathrm{~Hz}$

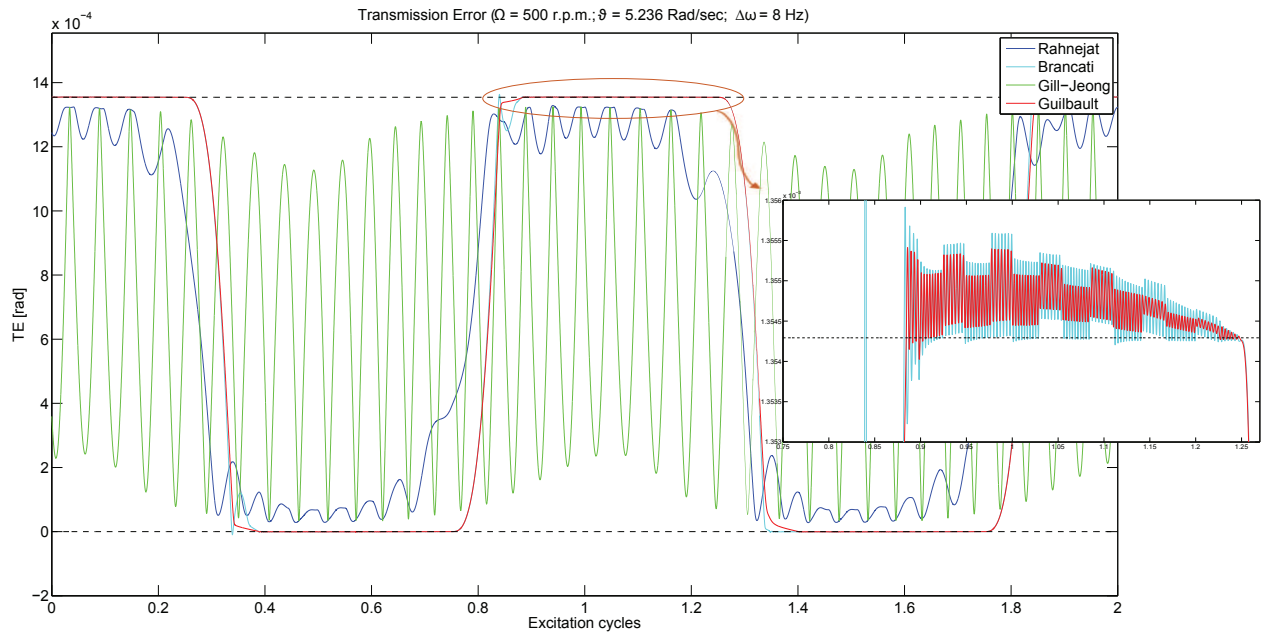

(b) Transmission error when $\Omega=500 \mathrm{rpm}, \vartheta=5.236 \mathrm{Rad} / \mathrm{s}$ and $\Delta \omega=8 \mathrm{~Hz}$

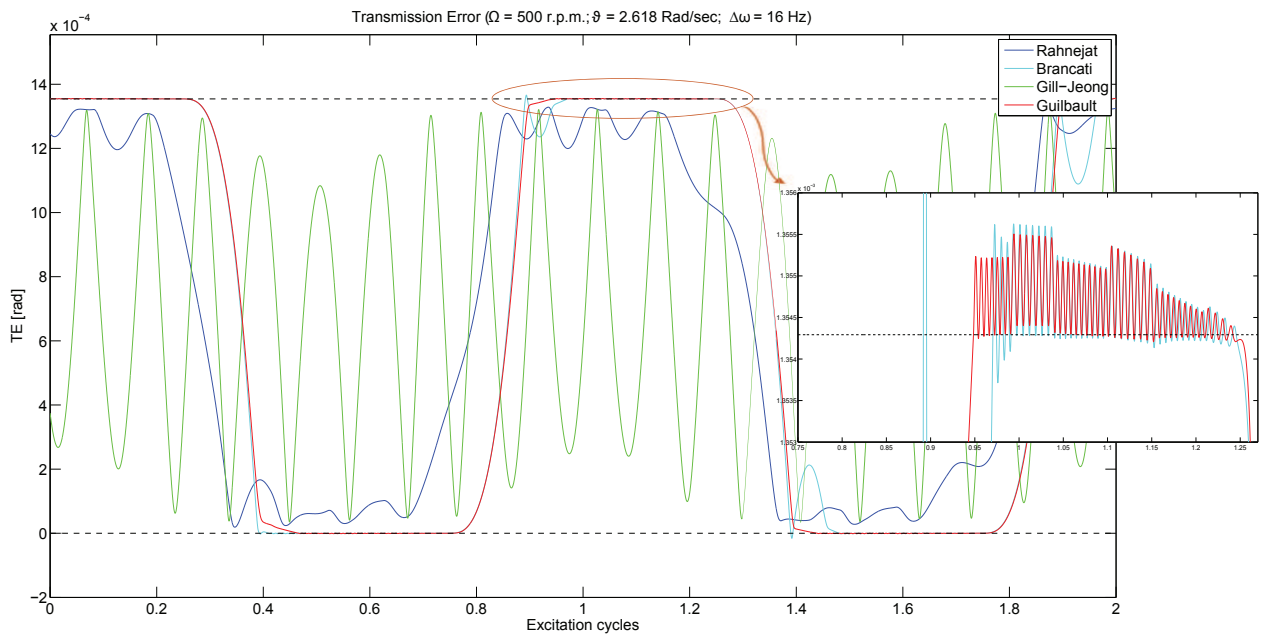

(c) Transmission error when $\Omega=500 \mathrm{rpm}, \vartheta=2.618 \mathrm{Rad} / \mathrm{s}$ and $\Delta \omega=16 \mathrm{~Hz}$

Fig. 11: Transmission error when mean velocity value is $500 \mathrm{rpm}$ for different harmonic excitations 


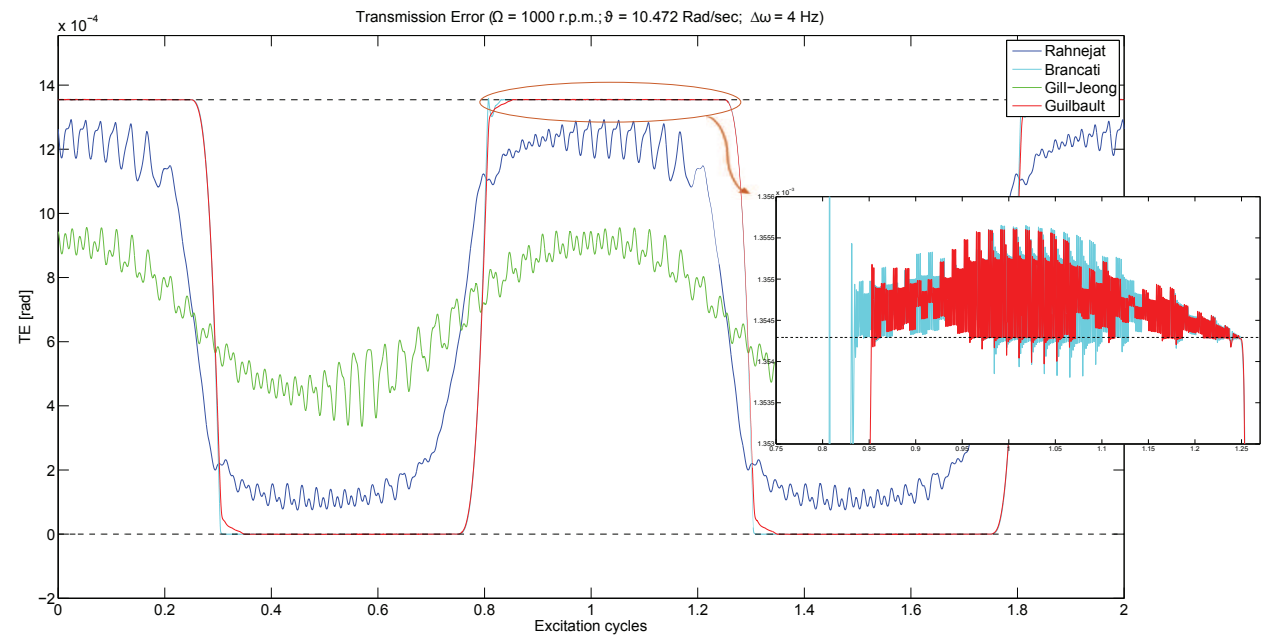

(a) Transmission error when $\Omega=1000 \mathrm{rpm}, \vartheta=10.472 \mathrm{Rad} / \mathrm{s}$ and $\Delta \omega=4 \mathrm{~Hz}$

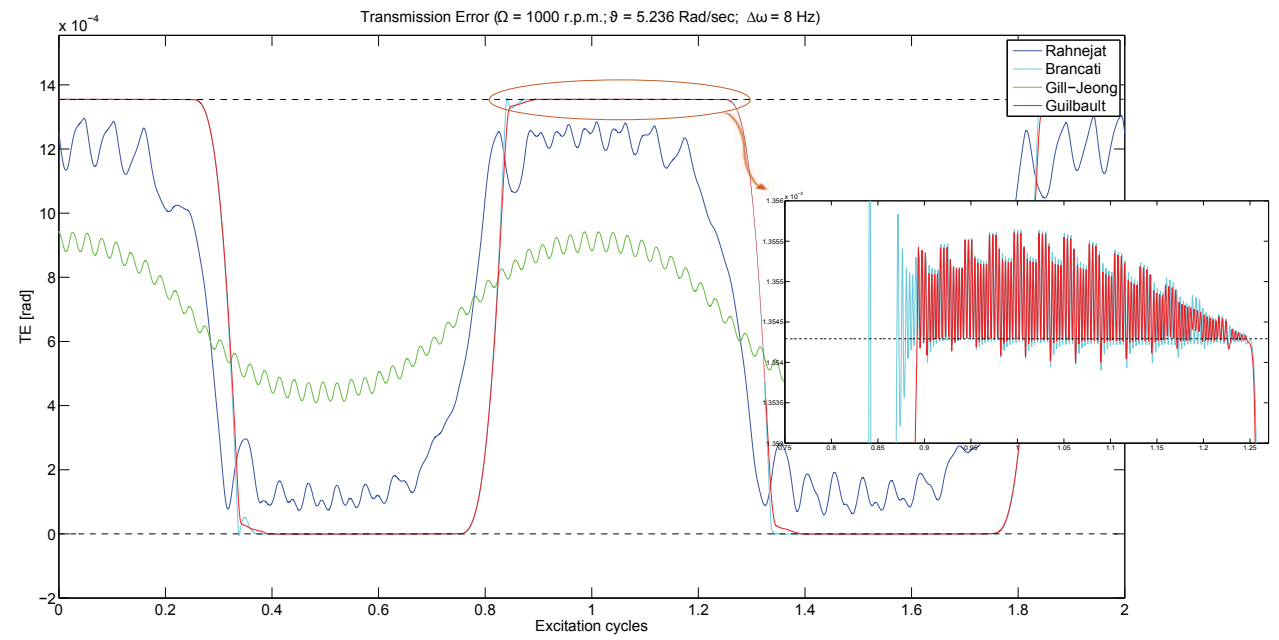

(b) Transmission error when $\Omega=1000 \mathrm{rpm}, \vartheta=5.236 \mathrm{Rad} / \mathrm{s}$ and $\Delta \omega=8 \mathrm{~Hz}$

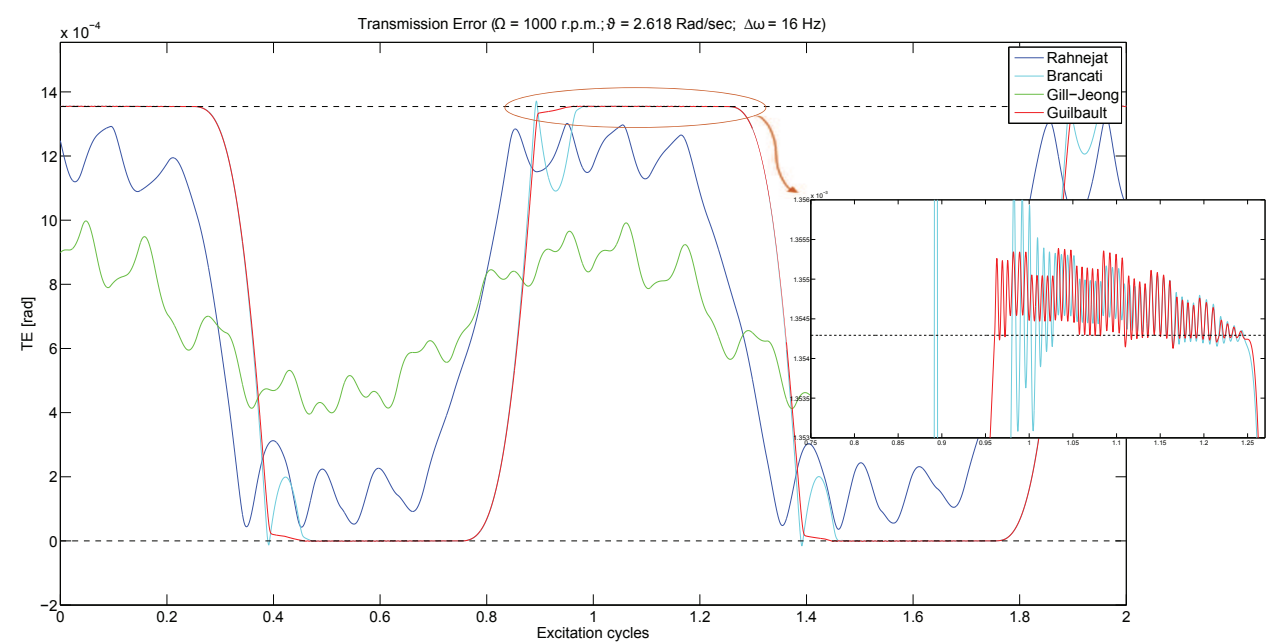

(c) Transmission error when $\Omega=1000 \mathrm{rpm}, \vartheta=2.618 \mathrm{Rad} / \mathrm{s}$ and $\Delta \omega=16 \mathrm{~Hz}$

Fig. 12: Transmission error when mean velocity value is $1000 \mathrm{rpm}$ for different harmonic excitations 
7. A. Diez-Ibarbia, A.F. del Rincon, M. Iglesias, A. de Juan, P. Garcia, and F. Viadero. Efficiency analysis of spur gears with a shifting profile. Meccanica, 51(3):707-723, 2016.

8. C.R. Evans and University of Cambridge. Measurement and Mapping of the Rheological Properties of Elastohydrodynamic Lubricants. University of Cambridge, 1983.

9. A. Fernández-del Rincón, M. Iglesias, A. de Juan, A. Diez-Ibarbia, P. García, and F. Viadero. Gear transmission dynamics: Effects of index and run out errors. Applied Acoustics, 2016.

10. A. Fernández del Rincón, M. Iglesias, A. De-Juan, P. García, R. Sancibrián, and F. Viadero. Gear transmission dynamic: Effects of tooth profile deviations and support flexibility. Applied Acoustics, 77(0):138-149, 32014.

11. A. Fernández Del Rincón, F. Viadero, M. Iglesias, P. García, A. De-Juan, and R. Sancibrian. A model for the study of meshing stiffness in spur gear transmissions. Mechanism and Machine Theory, 61:30-58, 2013.

12. P. Flores. Kinematics and dynamics of mechanical systems with lubricated revolute joints: The infinitely-short journalbearing approach. International Review of Civil Engineering, 6(5):117-123, 2015.

13. C. Gill-Jeong. Analysis of the nonlinear behavior of gear pairs considering hydrodynamic lubrication and sliding friction. Journal of Mechanical Science and Technology, 23(8):2125-2137, 2009.

14. R. Guilbault, S. Lalonde, and M. Thomas. Nonlinear damping calculation in cylindrical gear dynamic modeling. Journal of Sound and Vibration, 331(9):2110-2128, 2012.

15. M. Hammami, R. Martins, M.S. Abbes, M. Haddar, and J. Seabra. Axle gear oils: Tribological characterization under full film lubrication. Tribology International, 106:109 - 122, 2017.

16. M. Iglesias, A. Fernandez del Rincon, A. de Juan, A. Diez-Ibarbia, P. Garcia, and F. Viadero. Advanced model for the calculation of meshing forces in spur gear planetary transmissions. Meccanica, 50(7):1869-1894, 2015.

17. S. Li and A. Kahraman. A tribo-dynamic model of a spur gear pair. Journal of Sound and Vibration, 332(20):4963 4978, 2013.

18. F.H. Liu, S. Theodossiades, L.A. Bergman, A.F. Vakakis, and D.M. McFarland. Analytical characterization of damping in gear teeth dynamics under hydrodynamic conditions. Mechanism and Machine Theory, 94:141 - 147, 2015.

19. P. Marques, C. Fernandes, R. Martins, and J. Seabra. Efficiency of a gearbox lubricated with wind turbine gear oils. Tribology International, $71: 7-16,2014$.

20. E. Mucchi, G. Dalpiaz, and A. Fernandez Del Rincon. Elasto-dynamic analysis of a gear pump-part iv: Improvement in the pressure distribution modelling. Mechanical Systems and Signal Processing, 50-51:193-213, 2015.

21. E. Mucchi, A. Rivola, and G. Dalpiaz. Modelling dynamic behaviour and noise generation in gear pumps: Procedure and validation. Applied Acoustics, 77:99-111, 2014.

22. C. Nutakor, A. Kłodowski, J. Sopanen, A. Mikkola, and J.I. Pedrero. Planetary gear sets power loss modeling: Application to wind turbines. Tribology International, 105:42-54, 2017.

23. J.R. Ottewill, S.A. Neild, and R.E. Wilson. An investigation into the effect of tooth profile errors on gear rattle. Journal of Sound and Vibration, 329(17):3495-3506, 2010.

24. H. Rahnejat and R. Gohar. The vibrations of radial ball bearings. Proceedings of the Institution of Mechanical Engineers, Part C: Journal of Mechanical Engineering Science, 199(3):181-193, 1985.

25. R. Russo, R. Brancati, and E. Rocca. Experimental investigations about the influence of oil lubricant between teeth on the gear rattle phenomenon. Journal of Sound and Vibration, 321(3-5):647-661, 2009.

26. S. Theodossiades, M. De la Cruz, and H. Rahnejat. Prediction of airborne radiated noise from lightly loaded lubricated meshing gear teeth. Applied Acoustics, 100:79 - 86, 2015.

27. S. Theodossiades, O. Tangasawi, and H. Rahnejat. Gear teeth impacts in hydrodynamic conjunctions promoting idle gear rattle. Journal of Sound and Vibration, 303(3-5):632-658, 2007. 